Tropical Journal of Pharmaceutical Research October 2021; 20 (10): 2083-2088

ISSN: $1596-5996$ (print); 1596-9827 (electronic) (C) Pharmacotherapy Group, Faculty of Pharmacy, University of Benin, Benin City, 300001 Nigeria.

\title{
Resveratrol ameliorates necrotizing enterocolitis in neonatal rats through regulation of inflammatory and apoptotic pathways
}

\author{
Xiaodong Hu, Tao Chen, He Xiao, Wang Liu, Tingbing Xie, Lihe Zheng, Dan \\ Wang, Qi Ruan* \\ Department of General Surgery, Chengfei Hospital, Chengdu, Sichuan 610091, China
}

*For correspondence: Email: 3097317821@qq.com; Tel/Fax: +86 2887407832

Sent for review: 30 March 2021

Revised accepted: 22 September 2021

\begin{abstract}
Purpose: To determine the efficacy of resveratrol in mitigating necrotizing enterocolitis (NEC) in neonatal rats.

Methods: Necrotizing enterocolitis (NEC) was induced in neonatal rats using hypoxia and hypothermia. At the completion of treatment, the intestinal tissues of the rats were isolated for evaluation of various biochemical parameters.

Results: There was significant increase in the levels of proinflammatory cytokines (TNF- $\alpha$, IL- 6 and IL$1 \beta)$ and oxidative stress markers (MDA, xanthine oxidase and nitric oxide) in intestinal tissues of NEC rats $(p<0.05)$. However, resveratrol treatment led to significant decrease in the levels of cytokines and oxidative stress parameters, relative to the NEC group $(p<0.05)$. Furthermore, Western blotting results showed up-regulation in protein expressions of inflammatory cytokines in NEC rats. However, the protein expressions of inflammatory cytokines were down-regulated in the NEC rats on treatment with resveratrol. Moreover, resveratrol reversed the NEC-induced up-regulations of Bax and caspase-3, as well as NEC-mediated down-regulation of Bcl-2.

Conclusion: These results demonstrate that resveratrol mitigates NEC-induced intestinal damage in neonatal rats via anti-inflammatory, anti-apoptotic and antioxidant mechanisms of action. Therefore, resveratrol is a potential therapeutic agent for NEC.
\end{abstract}

Keywords: Resveratrol, Necrotizing enterocolitis, Neonate, Inflammation, Oxidative stress, Apoptosis

\begin{abstract}
This is an Open Access article that uses a funding model which does not charge readers or their institutions for access and distributed under the terms of the Creative Commons Attribution License (http://creativecommons.org/licenses/by/4.0) and the Budapest Open Access Initiative (http://www.budapestopenaccessinitiative.org/read), which permit unrestricted use, distribution, and reproduction in any medium, provided the original work is properly credited.
\end{abstract}

Tropical Journal of Pharmaceutical Research is indexed by Science Citation Index (SciSearch), Scopus, International Pharmaceutical Abstract, Chemical Abstracts, Embase, Index Copernicus, EBSCO, African Index Medicus, JournalSeek, Journal Citation Reports/Science Edition, Directory of Open Access Journals (DOAJ), African Journal Online, Bioline International, Open-J-Gate and Pharmacy Abstracts

\section{INTRODUCTION}

Necrotizing enterocolitis (NEC) is a serious inflammatory disease of the gastrointestinal system, and it is associated with significant morbidity and mortality. Necrotizing enterocolitis is one of major causes of neonatal intensive care admissions in hospitals. It has been reported that the global incidence of NEC ranges from 7 to $11 \%$ in newborns weighing < $1500 \mathrm{~g}$, or neonates of gestational age less than 28 weeks [1]. The clinical features of NEC are variable degrees of intestinal injury, sepsis due to bacterial invasion, and loss of organ functions 
[2]. The prime factors involved in the pathogenesis of NEC are low birth weight, prematurity, formula feeding, reduced oxygen supply, altered gut microflora, intestinal ischemia and necrosis, cytokines and poor antioxidant status [3]. Furthermore, reports suggest that reactive oxygen species (ROS) play crucial roles in the pathogenesis of NEC.

Studies have shown that the interaction between ROS generated as result of I/R injury to premature gut, and decreased antioxidant status, may lead to the development of NEC [4]. Thus, pathological factors such as immature antioxidant defences and increased ROS predispose to intestinal oxidative damage in premature newborns. Furthermore, increased levels of inflammatory cytokines, nitric oxide and toll-like receptor-4 activation have been implicated in the development of NEC [5]. Resveratrol is a major polyphenolic compound present in red grapes and wine beverages. It possesses antioxidant, anti-inflammatory, neuroprotective, anti-cancer and hepatoprotective properties [6]. Resveratrol has shown promising therapeutic effects in a wide range of neonatal pathologies. Previous studies have revealed the mitigative effect of resveratrol on hyperoxia-mediated lung injury, hypoxia/ischemia-provoked neuronal injury, and alcohol-induced neurotoxicity in neonatal rats [7]. Clinical and preclinical studies have reported the anti-inflammatory effect of resveratrol during inflammatory colitis [8]. The current research was carried out to investigate the protective effect of resveratrol against experimental NEC in neonatal rats.

\section{EXPERIMENTAL}

\section{Animals}

The present study was approved by the Animal Ethics Committee of Chengfei Hospital, Sichuan, China (approval no. SCH/19/008). The experimental procedure was performed as per the guidelines stated in the Care and Use of Laboratory Animals, NIH, USA [8]. Forty term, disease-free, neonatal Sprague-Dawley rats were used in this study. The neonatal rats were maintained under standard laboratory conditions at a mean temperature of $24 \pm 0.5^{\circ} \mathrm{C}$ and relative humidity of $50-55 \%$ humidity under 12-h light/12-h dark cycle.

\section{Establishment of neonatal model of NEC, and treatment with resveratrol}

The rats were randomly assigned to four groups: control, NEC, resveratrol-alone and resveratrol + NEC, with 10 rats in each group. In this study, NEC was induced using the hypoxia and hypothermia procedure as previously described by Karadag et al. The neonatal rats were made hypoxic by exposing them to $100 \%$ nitrogen gas for $2 \mathrm{~min}$, followed by instant exposure to cold stress at $4{ }^{\circ} \mathrm{C}$ for 10 min. The procedure was carried out two times daily for 3 days. Furthermore, the pups in resveratrol-alone and resveratrol + NEC groups received resveratrol at a dose of $15 \mathrm{mg} / \mathrm{kg}$ twice daily for 3 days, $30 \mathrm{~min}$ before induction of NEC. The body weights of the animals were recorded daily. After 4 days, the neonatal rats were euthanized via cervical dislocation. Following sacrifice, the intestines of the neonatal rats were examined for NEC-induced pathological changes such as edema, fragility, discoloration, perforation, and necrosis. From each neonatal rat, $3 \mathrm{~cm}$ of the ileum at the terminal region encompassing the cecum, was isolated for the estimation of various disease markers. The excised tissues were rinsed in icecold saline, after which $50 \%$ of the tissues were used for preparation homogenates for biochemical analysis. The remaining tissues were preserved in liquid nitrogen at $-70^{\circ} \mathrm{C}$ for western blotting studies.

\section{Biochemical analysis}

\section{Proinflammatory cytokines}

The intestinal levels of TNF- $\alpha$, IL- 6 and IL- $1 \beta$ were assayed using enzyme-linked immunosorbent assay (ELISA) kits (Sigma, USA) in accordance with the manufacturer's instructions.

\section{Oxidative stress indexes}

The levels of malondialdehyde (MDA) and xanthine oxidase $(\mathrm{XO})$ in intestinal tissue were determined using assay kits (Nanjing Jiancheng Bioengineering Institute, Nanjing, Jiangsu, China).

\section{Antioxidant parameters}

The intestinal tissue levels of SOD, CAT, GPx and GSH were assayed using appropriate assay kits procured from Nanjing Jiancheng Bioengineering Institute, Nanjing, Jiangsu, China.

\section{Nitrite levels}

Nitrite (NO) levels in the intestinal homogenate were measured using the procedure of Green et al. Equal volumes of tissue homogenate and the 
Greiss reagent were mixed and shaken. Then, the resultant solution was kept in dark for 10 min at $37^{\circ} \mathrm{C}$, after which the absorbance was read at $540 \mathrm{~nm}$ in a UV- spectrophotometer. The concentration of nitrite was expressed as $\mu \mathrm{mole} / \mathrm{g}$ of tissue.

\section{Western blot analysis}

Total protein was extracted from the intestinal tissue samples using lysate solution $(200 \mu \mathrm{L})$ containing protease inhibitor (Beyotime, Hunan, China), and the lysate was kept in ice-cold conditions for $20 \mathrm{~min}$, prior to centrifugation at 4 ${ }^{\circ} \mathrm{C}$ at $13,000 \mathrm{rpm}$ for $30 \mathrm{~min}$. The protein concentration of the lysate was determined using BCA method. Then, equal amounts of proteins were subjected to SDS-polyacrylamide gel electrophoresis, followed by transfer onto PVDF membranes (Millipore, Bedford, MA, USA). The PVDF membranes were blocked in 5 $\%$ bovine serum albumin at $37^{\circ} \mathrm{C}$ for $2 \mathrm{~h}$. Thereafter, the PVDF membranes were incubated overnight at $4{ }^{\circ} \mathrm{C}$ with the primary antibodies: rabbit anti-TNF- $\alpha$, rabbit anti-IL-6, rabbit anti-IL-1 $\beta$, rabbit anti- $\mathrm{BCl}-2$, anti-Bax, anti-caspase-3, and rat anti-GAPDH. This was followed by incubation with secondary antibody for $2 \mathrm{~h}$ at $37{ }^{\circ} \mathrm{C}$. The protein expressions were observed through ECL kit. The internal control was GAPDH.

\section{RESULTS}

Effect of resveratrol on tissue inflammatory cytokine concentrations in NEC neonatal rats

The intestinal levels of TNF- $\alpha$, IL -6 and IL-1 $\beta$ were markedly higher in NEC group than in the control group ( $p<0.05)$. However, treatment of the NEC pups with resveratrol led to significant decreases in levels of TNF- $\alpha$, IL- 6 and IL-1 $\beta$, when compared to untreated NEC group ( $p<$ 0.05).

\section{Effect of resveratrol on intestinal levels of MDA and xanthine oxidase in NEC neonatal rats}

The levels of oxidative stress markers i.e., malondialdehyde (MDA) and xanthine oxidase (XO) were significantly increased in the intestinal homogenate of NEC neonatal rats $(p<$ 0.05; Figure 1). However, treatment with resveratrol significantly reduced the oxidative stress by reducing the levels of MDA and XO ( $p$ $<0.05$ )

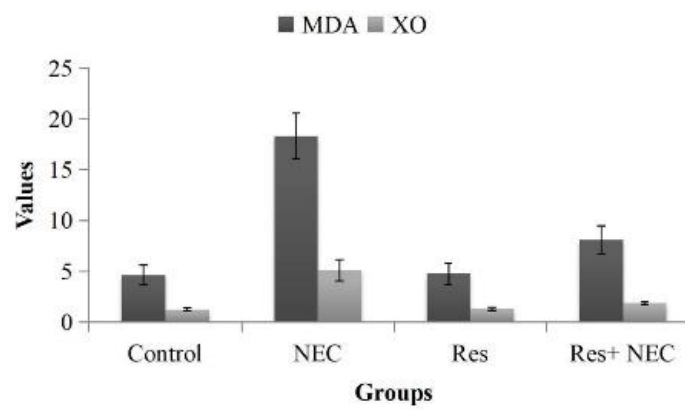

Figure 1: Effect of resveratrol on intestinal MDA and $X O$ levels in NEC neonatal rats. The NEC neonatal rat intestinal homogenates were assayed for MDA and XO levels after resveratrol treatment. MDA: malondialdehyde (nmol/g protein); XO: xanthine oxidase (U/mg protein)

\section{Effect of resveratrol on intestinal levels of antioxidants in NEC neonatal rats}

The intestinal contents of SOD, CAT, GPx and GSH were markedly reduced in NEC neonatal rats, when compared to the control rat pups ( $p<$ 0.05). However, resveratrol administration to NEC rat pups effectively reversed the decreases in the levels of these parameters. These results are shown in Table 2.

Table 1: Effect of resveratrol on intestinal cytokine levels in NEC rats

\begin{tabular}{lccc}
\hline Group & TNF- $\alpha$ (pg/mg protein) & IL-6 (pg/mg protein) & IL-1 $\beta$ (pg/mg protein) \\
\hline Control & $55.76 \pm 6.65$ & $62.24 \pm 7.25$ & $21.48 \pm 2.87$ \\
NEC & $109.76 \pm 9.87^{\mathrm{a}^{*}}$ & $123.54 \pm 12.34^{\mathrm{a}^{*}}$ & $40.12 \pm 5.18^{\mathrm{a}^{*}}$ \\
Res & $58.45 \pm 8.58$ & $65.15 \pm 6.42$ & $22.75 \pm 2.12$ \\
Res + NEC & $75.15 \pm 9.24^{\mathrm{b}^{*}}$ & $81.24 \pm 10.95^{\mathrm{b}^{*}}$ & $28.21 \pm 3.18^{\mathrm{b}^{*}}$ \\
\hline (Res $=$ resveratrol) & & &
\end{tabular}

Table 2: Effect of resveratrol on intestinal levels of SOD, CAT, GPx and GSH in NEC rats

\begin{tabular}{lccc}
\hline Group & SOD (U/mg protein) & CAT (U/mg protein) & GPx (U/mg protein) \\
\hline Control & $4.25 \pm 0.75$ & $14.56 \pm 3.12$ & $95.45 \pm 10.56$ \\
NEC & $1.86 \pm 0.09^{\mathrm{a}^{*}}$ & $8.28 \pm 1.56 \mathrm{a}^{\mathrm{a}^{*}}$ & $\frac{52.76 \pm 4.53^{\mathrm{a}^{*}} 94.72 \pm 9.12}{86.42 \pm 7.32^{\mathrm{b}^{*}}}$ \\
Res & $4.12 \pm 0.85$ & $14.12 \pm 3.76$ & $86.42 \pm 5^{\mathrm{b}^{*}}$ \\
Res + NEC & $3.86 \pm 0.5 \mathrm{~b}^{*}$ & $13.24 \pm 3.0 \mathrm{~b}^{*}$ & \\
\hline
\end{tabular}




\section{Effect of resveratrol on intestinal levels of} nitric oxide in NEC neonatal rats

The levels of nitric oxide (NO) were markedly ( $p$ $<0.05$ ) increased in the intestinal homogenate of NEC neonatal rats, when compared to normal rats (Figure 2). However, treatment with resveratrol significantly $(p<0.05)$ reduced the NO levels.

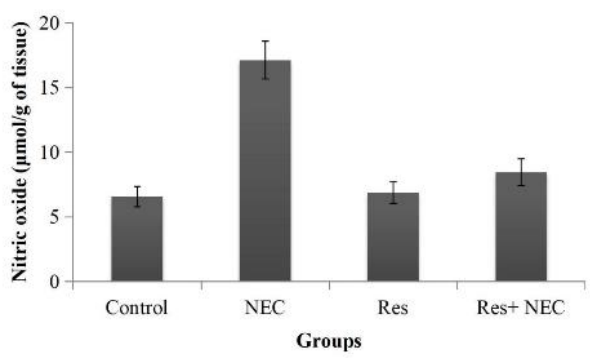

Figure 2: Effect of resveratrol on intestinal $\mathrm{NO}$ levels in NEC neonatal rats. The NEC neonatal rats showed increased intestinal NO levels. However, resveratrol treatment effectively reduced the $\mathrm{NO}$ levels

Effect of resveratrol on protein expressions of inflammatory cytokines and apoptotic markers

The NEC neonatal rats showed markedly higher degree of inflammation which was evident in increased expression levels of TNF- $\alpha$, IL- 6 and $\mathrm{IL}-1 \beta$, relative to the control rats (Figure 3 ). The relative protein level of inflammatory cytokines was also higher in NEC neonatal rats than in control rats. Furthermore, the protein level of apoptotic marker, Bcl-2 was downregulated, while Bax protein and caspase-3 protein were upregulated in NEC neonatal rats, when compared to control rats. However, resveratrol administration significantly reversed the NECinduced changes in proinflammatory and apoptotic proteins in the neonatal rats.

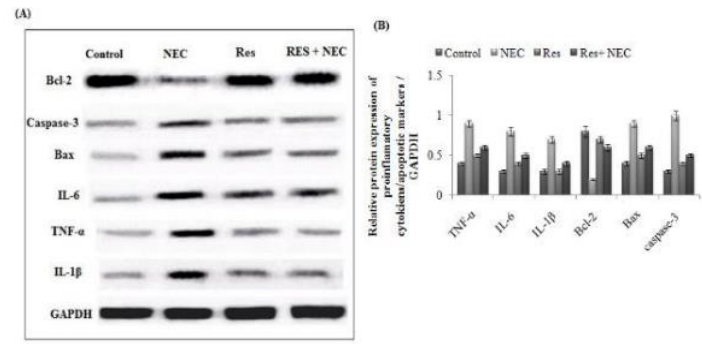

Figure 3: Effect of resveratrol on protein expression levels of inflammatory cytokines and apoptotic markers. (A) Western blot analysis of inflammatory and apoptotic markers in NEC neonatal rats. (B) Relative protein expression levels of proinflammatory cytokines/apoptotic markers

\section{DISCUSSION}

Immature intestine, genetic factors, loss of microvascular structure, alteration in intestinal microflora, immune-compromised intestine and formula feeding are the hallmark pathological factors involved in altered immune response seen in NEC. An increase in intestinal inflammation is associated with differentiation of neonates from the mature intestine [9]. The current research has demonstrated that resveratrol might be a suitable candidate drug for ameliorating altered immunity manifested in neonatal murine model of hypoxia/hypothermiainduced NEC.

Exposure of the intestinal tissues to ischemia and hypoxia conditions triggers proinflammatory processes and increases cytokine secretion, free radical generation and neutrophil activation [10]. The released free radicals damage vital molecules such as DNA, cell lipids and proteins, leading to tissue injury and NEC [11]. In the present study, the level of MDA, an accepted marker of oxidative stress-mediated lipid peroxidation, was increased in intestinal tissue of NCE neonatal rats [12]. The intestinal hypoxia seen during NEC is caused by hydroxyl free radicals produced in xanthine oxidase-mediated reactions. The present study found elevated activity of xanthine oxidase in intestinal homogenate of NEC neonatal rats, which is consistent with earlier reports [13]. The increased levels of MDA and XO in NEC neonatal rats were significantly reduced by resveratrol treatment. It is known that the inactivation of xanthine oxidase by resveratrol blocks the synthesis of hypoxanthine and ROS during ischemia. The free radical-scavenging property of resveratrol is due to the presence of a phenolic moiety and three free hydroxyl groups which neutralize the free radicals generated during ischemic conditions.

In normal intestines, the free radicals are generated by enzymic and non-enzymic antioxidants such as SOD, CAT, GPx and reduced glutathione. However, in the case of intestinal damage due to NEC-induced oxidative stress, the intestinal membrane integrity is compromised, leading to reduced antioxidant defence which is the hallmark for intestinal tissue damage in NEC in neonates [14]. Similarly, the present study observed decreased concentrations of antioxidants (SOD, CAT, and GPx) in intestinal tissues of NEC neonatal rats. However, resveratrol treatment significantly increased the intestinal tissue levels of SOD, CAT and GPx, thereby reducing the NECinduced oxidative stress in neonatal rats. Thus, 
the present study has demonstrated that resveratrol significantly increased antioxidant levels during intestinal I/R injury, which is consistent with previous findings [14].

Nitric oxide (NO) is a moderately reactive radical produced from L-arginine by the catalytic action of nitric oxide synthase (NOS). The three isoforms of NOS are nNOS (constitutive and neuronal), iNOS (inducible) and eNOS (endothelial). Previous reports indicate that iNOS expression is upregulated in pathological conditions such as endotoxemia and septic shock, leading to tissue damage and gut barrier dysfunction [15]. Furthermore, RT-PCR studies have revealed elevated iNOS expressions in intestines of neonates diagnosed with NEC [15]. This indicates that increased expression of iNOS increases the production of $\mathrm{NO}$ and reactive nitrogen free radicals, thereby aggravating gut barrier dysfunction in NEC. In this study, increased levels of $\mathrm{NO}$ in intestinal tissue homogenates of NEC were seen in neonatal rats. However, resveratrol treatment significantly reduced the $\mathrm{NO}$ levels to normal values. This is in agreement with the results obtained in a study by Ozkan et al who reported marked reductions in $\mathrm{NO}$ levels in intestinal I/R rats after treatment with resveratrol [16].

The pro-inflammatory cytokines are crucial in the progression of NEC. They exert their effects through epithelial barrier destruction, thereby aggravating intestinal injury [17]. When compared with other pro-inflammatory cytokines, TNF- $\alpha$ plays a very predominant role in the pathogenesis of NEC [17]. Previous studies reported increased concentrations of TNF- $\alpha$ in hepatic tissue and intestine of NEC rats [18]. The precise mechanism through which TNF-a participates in the progression of NEC is still obscure. However, reports suggest that TNF-a increases the production of metalloproteinase stromelysin-l which is involved in matrix degradation and tissue damage. Furthermore, TNF- $\alpha$ is involved in triggering various cytokines and apoptosis in intestinal tissues, and in enhancement of the production of free radicals at the site of inflammation [18].

Loss of intestinal barrier permeability leads to increased release of the pro-inflammatory cytokine, IL- $1 \beta$ and inflamed intestines [19]. In addition, IL- $1 \beta$ is an important inflammatory mediator which is actively involved in immune response during NEC [19]. Interleukin-6 (IL-6) is another cytokine which increases neutrophil recruitment, causes rampant release of oxygenderived free radical at the site of inflamed intestine, and causes cytotoxicity [20]. In the present study, there were elevated levels of intestinal TNF- $\alpha, \mathrm{IL}-1 \beta$ and IL- 6 in NEC neonatal rats. Furthermore, upregulated protein expressions of TNF- $\alpha$, IL- $1 \beta$ and IL- 6 were seen in NEC neonatal rats. However, resveratrol treatment significantly downregulated the protein expression levels of TNF- $\alpha$, IL- $1 \beta$ and IL- 6 in NEC rats through its anti-inflammatory and antioxidant effects [21].

Intestinal apoptosis is a predominant pathological feature in preterm infants, due to NEC [22]. It is known that $\mathrm{Bcl}-2$ and related proteins are vital biomolecules involved in apoptosis-regulatory processes. Moreover, the equilibrium between pro- and anti-apoptotic molecules is important for cellular activity [22]. The 'apoptotic rheostat' Bcl2/Bax is important in the regulation of apoptosis which also controls cellular flux towards or away from apoptosis [22]. Furthermore, intestinal tissue Bax-to-Bcl-2 ratio is widely employed as a reliable marker for evaluating responses to proand anti-apoptotic insults [23]. In this study, NEC neonatal rats had upregulated and downregulated protein expressions of Bax and $\mathrm{Bcl}-2$, respectively, indicating marked apoptosis in the intestinal tissue. A previous report showed that caspase-3, a unique pro-apoptosis marker, was up-regulated in the intestinal tissue of NEC rats [23]. In this study, the NEC neonatal rats also showed increased protein expression level of caspase-3 in intestinal tissue, which confirmed apoptosis. The anti-apoptotic efficacy of resveratrol in hypoxia and ischemic conditions have been reported in previous studies [23].

\section{CONCLUSION}

The present study has demonstrated that resveratrol exerted potential protective effect against NEC in the neonatal rat model. Resveratrol mediated its effect through antioxidative, anti-inflammatory and anti-apoptotic mechanisms. Therefore, resveratrol may be used as potential therapeutic agent for NEC.

\section{DECLARATIONS}

\section{Conflict of Interest}

No conflict of interest associated with this work.

\section{Contribution of Authors}

Authors declare that this is their own work and all liabilities pertaining to claims relating to the content of this article will be borne by the authors. Qi Ruan - conceived, designed the study and wrote the manuscript. Xiaodong $\mathrm{Hu}$, 
Tao Chen, He Xiao, Wang Liu, Tingbing Xie, Lihe Zheng and Dan Wang- collected, analysed the data and made the literature survey. All authors approved the manuscript for publication.

\section{Open Access}

This is an Open Access article that uses a funding model which does not charge readers or their institutions for access and distributed under the terms of the Creative Commons Attribution License (http://creativecommons.org/licenses/by/ 4.0) and the Budapest Open Access Initiative (http://www.budapestopenaccessinitiative.org/rea d), which permit unrestricted use, distribution, and reproduction in any medium, provided the original work is properly credited.

\section{REFERENCES}

1. Qian $T$, Zhang R, Zhu L, Shi P, Yang J, Yang $C Y$, Chen $D M$. Necrotizing enterocolitis in low birth weight infants in China: Mortality risk factors expressed by birth weight categories Pediatr Neonatol 2017; 58(6): 509-515.

2. Thompson AM, Bizzarro MJ. Necrotizing enterocolitis in newborns: pathogenesis, prevention and management. Drugs 2008; 68(9): 1227-38

3. Alganabi M, Lee C, Bindi E, Li B, Pierro A. Recent advances in understanding necrotizing enterocolitis. F1000Res. 2019; 8: F1000 Faculty Rev-107.

4. Kelly N, Friend $K$, Boyle P. The role of the glutathione antioxidant system in gut barrier failure in a rodent model of experimental necrotizing enterocolitis. Surgery 2004; 136(3): 557-566

5. Viscardi RM, Lyon NH, Sun CC, Hebel JR, Hasday JD. Inflammatory cytokine mRNAs in surgical specimens of necrotizing enterocolitis and normal newborn intestine. Pediatr Pathol Lab Med 1997; 17(4): 547-559

6. Salehi B, Mishra AP, Nigam M. Resveratrol: A DoubleEdged Sword in Health Benefits. Biomedicines 2018 ;6(3): 91.

7. Bian $H$, Shan $H$, Chen $T$. Resveratrol ameliorates hypoxia/ischemia-induced brain injury in the neonatal rat via the miR-96/Bax axis. Childs Nerv Syst. 2017; 33(11): 1937-1945

8. Alrafas $H R$, Busbee PB, Nagarkatti M, Nagarkatti PS. Resveratrol modulates the gut microbiota to prevent murine colitis development through induction of Tregs and suppression of Th17 cells. J Leukoc Biol 2019; 106(2): 467-480.

9. Karadag A, Ozdemir R, Kurt A. Protective effects of Dexpanthenol in an experimental model of necrotizing enterocolitis. J Pediatr Surg 2015; 50(07): 1119-1124
10. Markel TA, Crisostomo PR, Wairiuko GM, Pitcher J, Tsai $B M$, Meldrum DR. Cytokines in necrotizing enterocolitis. Shock 2006; 25: 329.

11. C. Aydemir, D. Dilli, N. Uras. "Total oxidant status and oxidative stress are increased in infants with necrotizing enterocolitis," Journal of Pediatric Surgery 2011; 46(11): 2096-2100.

12. Harpavat S, Pammi M, Gilger M. Novel treatments for NEC: Keeping IBD in mind. Curr. Gastroenterol. Rep 2012; 14: 373-379

13. Mozaffari S, Nikfar S. A Review on the Role of Oxidative Stress and Inflammation in Necrotizing Enterocolitis and Benefits of the Phosphodiesterase Inhibitor Pentoxifylline. Int. J Pharmacol 2013; 9: 245-250

14. Hsueh W, Caplan MS, Qu XW, Tan XD, De Plaen IG, Gonzalez- Crussi F. Neonatal necrotizing enterocolitis: clinical considerations and pathogenetic concepts. Pediatr Dev Pathol 2003; 6: 6.

15. Potoka DA, Nadler EP, Upperman JS. Role of nitric oxide and peroxinitrite in gut barrier failure. World $J$ Surg 2002; 26: 806-11.

16. Ozkan OV, Yuzbasioglu MF, Ciralik H, Kurutas EB, Yonden Z, Aydin M, Bulbuloglu E. Resveratrol, a natural antioxidant, attenuates intestinal ischemia/reperfusion injury in rats. Tohoku J Exp Med 2009; 218(3): 251-8.

17. Markel TA, Crisostomo PR, Wairiuko GM, Pitcher J, Tsai $B M$, Meldrum DR. Cytokines in necrotizing enterocolitis. Shock 2006; 25: 329.

18. Zhang BH, Yu HG, Sheng ZX. The therapeutic effect of recombinant human trefoil factor 3 on hypoxia-induced necrotizing enterocolitis in immature rats. Regul Pept 2003; 116(1-3): 53 - 60.

19. Hering NA, Fromm M, Schulzke JD. Determinants of colonic barrier function in inflammatory bowel disease and potential therapeutics. J Physiol 2012; 590: 1035

20. Qi W, Shen Q, Zhang L, Han LP, Wang S. Study on the inflammatory intervention of erythropoietin on NEC. Exp Ther Med 2016; 11: 2221-2224.

21. Parlar A, Arslan SO. Resveratrol Normalizes the Deterioration of Smooth Muscle Contractility after Intestinal Ischemia and Reperfusion in Rats Associated with an Antioxidative Effect and Modulating Tumor Necrosis Factor Alpha Activity. Ann Vasc Surg 2019; 61: 416-426.

22. Huang LG, Zhou W, Rong X, Tao L, Lu WN. Effects of glycomacropeptide on damage to intestinal tissue and apoptosis of intestinal epithelial cells in neonatal rats with necrotizing enterocolitis. Zhonghua Er Ke Za Zhi. 2012; 50: 536-542.

23. Clark JA, Doelle SM, Halpern MD, Saunders TA, Holubec $H$, Dvorak K, Boitano SA, Dvorak B. Intestinal barrier failure during experimental necrotizing enterocolitis: protective effect of EGF treatment. Am. J. Physiol. Gastrointest. Liver. Physiol 2006; 291: G938-949. 